\title{
Group Invariant Solutions and Local Conservation Laws of Heat Conduction Equation Arising Laser Heating Carbon Nanotubes Using Lie Group Analysis
}

\author{
Emrullah Yaşar* and Yakup Yıldırım
}

\begin{abstract}
In this study, based on the continuous transformations of Lie groups, the exact analytic solutions of the laser heating carbon nanotubes formulated by using the classical heat conduction equation with various physical properties were constructed. These solutions are the type of group invariant solutions. The constructed solutions have expanded and enriched the solution forms of this new model existing in the literature. With the help of the Maple package program, 3D, density, and contour graphs were drawn for the special values of the parameters in the solutions, and the physical structures of the solutions obtained in this way were also observed. The solutions obtained can be used in the explanation of physical phenomena occurring in cancer investigations.
\end{abstract}

Keywords: Carbon nanotubes; Lie groups; Conservation laws.

AMS Subject Classification (2020): Primary: 37L20; 35C05 ; Secondary: 37K06; 34A05

${ }^{*}$ Corresponding author

\section{Introduction}

It is well known that the evolution differential equations (EDEs) mathematically model many physical phenomena that occur in nature. Many analytical and numerical solution techniques such as Hirota bilinear method, Backlund transformations, Darboux transformations, Painleve property, variational iteration method, tanh method, invariant subspace method, Lie symmetry groups etc. have been developed over time to solve those equations [1-14]. Among the methods listed above, the Lie symmetry groups method is an effective approach in obtaining exact solutions (special group invariant solutions) of the considered differential equations (systems), regardless of the order, degree and linearity types [14, 15].

Carbon nanotubes (CNTs) have an important place in nanomaterials science due to their mechanical, electrical, optical and magnetic properties. CNTs have effective applications in the field of medicine, drug distribution, and contrast agents. One of the physical applications that these EDEs address is cancer disease. We know from experimental studies that CNTs are promising nanomaterials for warming agents in photothermal therapy (PTT) 
and contrast agents in photoacoustic (PA) imaging. In the experiments, the temperature of the agents used in both PTT and PA imaging during laser irradiation was examined in the tissue. It is also known from experimental studies that cancer cells can be destroyed by increasing the temperature in the tissue (with the help of the agents in PTT) to 41-47 ${ }^{\circ} \mathrm{C}$. Thus, cancer cells become hyperthermic and suffer significant damage [16].

When we look at the studies in the literature, in the study [17], the heat analysis of multi-walled CNTs during pulsed laser heating was investigated using the finite element method (FEM) for the classical heat conduction equation. The dynamics of pulsed nanosecond laser heating process was simulated by the solution of the heat conduction equation. In addition, the FEM is applied to compute the temperature profiles as a function of depth $x$ and time $t$ in the sample (multi-walled CNT) [18]. Also, in the literature optical soliton-like solutions for the system of ring-cavity fiber laser using carbon nanotubes for passive mode locking have also been studied [19]. In [20], the authors investigated more information on the system of carbon nanotubes conveying fluid by using Lie symmetry groups.

In this study, we examine the temperature profiles using the Lie symmetry groups method and obtain analytical solutions, based on the classical heat conduction equation that explains the laser-heated CNT model previously discussed in the study [16]. In the study [16], the authors considered the temperature function as dependent only on the radial variable and treated the model as a simple ordinary differential equation (ODE). Besides, we will try to obtain the conservation laws of the model.

The study is organized as follows: The laser heated governing model discussed in Section 2 is presented. The solution of the model will be explored in Section 3. Section 4 is devoted to the conservation laws of the model through the multiplier method. The results and remarks are presented in Section 5.

\section{Governing equation}

We will examine the CNT, which is exposed to laser heating and has a cylindrical structure. Here we will assume that there are cancerous tissues around the CNT. Besides, it will be assumed that the length of the CNT is greater than its radius, and the temperature $T_{b}$ is 37 degrees at a unit distance $b$ from the center. Considering the above-mentioned assumptions, CNTs exposed to laser heating can be formulated with the heat conduction equation given below:

$$
k_{1} k_{2} u_{t}=k_{3} \frac{1}{x}\left(x u_{x}\right)_{x}+f(x, t), \quad 0<x<a,
$$

where $k_{1}$ is the density of CNT, $k_{2}$ is the concentration of CNT, $k_{3}$ is the thermal conductivity of CNTs and $u(x, t)$ and $f(x, t)$ denote the temperature function and source term respectively, where $x$ is the distance measured from the center of the cylinder and $t$ is the time variable. In our work we will assume that $f(x, t)$ is a constant let say $f$ (in fact, we learn from [16] that based on the physical meaning of $f(x, t)$, its mathematical formulation is in the form of $f(x, t)=(1-R) I_{0} \alpha$ where $R$ denotes reflectivity, $I_{0}$ is the laser intensity, $\alpha$ is optical absorption coefficient of CNTs) (see, [16] for further details).

\section{Lie point symmetries of Eq.(2.1)}

Now consider the continuous Lie transformations with one small parameter given below:

$$
\begin{aligned}
\bar{x} & =x+\mu \xi(t, x, u)+O(\mu)^{2}, \\
\bar{t} & =t+\mu \tau(t, x, u)+O(\mu)^{2}, \\
\bar{u} & =u+\mu \eta(t, x, u)+O(\mu)^{2} .
\end{aligned}
$$

In this case, the Lie point symmetry generator of Eq. (2.1) associated with (3.1) is generated by the vector field of the form

$$
X=\tau(t, x, u) \frac{\partial}{\partial t}+\xi(t, x, u) \frac{\partial}{\partial x}+\eta(t, x, u) \frac{\partial}{\partial u},
$$

where $\tau, \xi$ and $\eta$ depend on only $t, x$ and $u$. Applying the second prolongation $p r^{(2)} X$

$$
p r^{(2)} X=X+\eta^{t} \frac{\partial}{\partial u_{t}}+\eta^{x} \frac{\partial}{\partial u_{x}}+\eta^{x x} \frac{\partial}{\partial u_{x x}},
$$

to Eq. (2.1), i.e,

$$
\left.p r^{(2)} X\left[k_{1} k_{2} u_{t}-k_{3} \frac{1}{x}\left(x u_{x}\right)_{x}-f\right]\right|_{k_{1} k_{2} u_{t}=k_{3} \frac{1}{x}\left(x u_{x}\right)_{x}+f}=0,
$$


we find that the coefficient functions $\xi(x, t, u), \tau(x, t, u)$ and $\eta(x, t, u)$ must satisfy the following linearized symmetry condition

$$
k_{1} k_{2} \eta^{t}+k_{3} \frac{1}{x^{2}} u_{x} \xi-k_{3} \frac{1}{x} \eta^{x}-k_{3} \eta^{x x}=0,
$$

where $\eta^{t}, \eta^{x}$, and $\eta^{x x}$ are the extended coefficients of $p r^{(2)} X$. In a simplified form these coefficients can be written in the following format:

$$
\begin{aligned}
\eta^{t} & =D_{t} \eta-u_{x} D_{t} \xi-u_{t} D_{t} \tau \\
\eta^{x} & =D_{x} \eta-u_{x} D_{x} \xi-u_{t} D_{x} \tau \\
\eta^{x x} & =D_{x}^{2} \eta-u_{x} D_{x}^{2} \xi-u_{t} D_{t}^{2} \tau-2 u_{x x} D_{x} \xi-2 u_{x t} D_{x} \tau
\end{aligned}
$$

where $D_{x}, D_{t}$ are the total derivatives with respect to $x$ and $t$, respectively and are given as follows:

$$
\begin{aligned}
D_{x} & =\frac{\partial}{\partial x}+u_{x} \frac{\partial}{\partial u}+u_{x t} \frac{\partial}{\partial u_{t}}+u_{x x} \frac{\partial}{\partial u_{x}}+\ldots . . \\
D_{t} & =\frac{\partial}{\partial t}+u_{t} \frac{\partial}{\partial u}+u_{x t} \frac{\partial}{\partial u_{x}}+u_{t t} \frac{\partial}{\partial u_{t}}+\ldots . .
\end{aligned}
$$

Here, we would like to point out that, for a differential equation (or system) of order $n$, the coefficient functions of $\operatorname{pr}^{(n)}(X)$ prolongation is given as the follows:

$$
\eta_{i_{1} \ldots i_{s}}^{\alpha}=D_{i_{s}}\left(\eta_{i_{1} \ldots i_{s-1}}^{\alpha}\right)-u_{j i_{1} \ldots i_{s-1}} D_{i_{s}}\left(\xi^{j}\right), s>1 .
$$

If we write the prolonged coefficients (3.6) in the linearized invariance condition (3.5) and equal the coefficients of the derivatives of $u$ with respect to $x$ to zero, we arrive at the following linear over-determined partial differential equation system:

$$
\begin{aligned}
\eta_{u u} & =0, \\
\eta_{x u} & =-\frac{\tau_{t t} k_{1} k_{2} x}{4 k_{3}}, \\
\eta_{x x} & =\frac{x k_{2} k_{1} \eta_{t}-f x \eta_{u}+f x \tau_{t}-k_{3} \eta_{x}}{k_{3} x}, \\
\eta_{t u} & =-\frac{\tau_{t t}}{2}, \\
\tau_{u} & =0 \\
\tau_{x} & =0, \\
\tau_{t t t} & =0, \\
\xi & =\frac{x \tau_{t}}{2} .
\end{aligned}
$$

If the above over-determined system of equations is solved for $\xi(x, t), \tau(t)$ and $\eta(x, t, u)$, the following useful and important Lie vector fields are obtained (in fact, 6 dimensional Lie vector algebras are obtained but some trivial vector fields are omitted). Hence, the point symmetry generators admitted by the heat equation (2.1) are given by

$$
\begin{aligned}
X & =\frac{\partial}{\partial t}+\left(\frac{x^{2}}{4}+\frac{k_{3} t}{k_{1} k_{2}}\right) \frac{\partial}{\partial u}, \\
Y & =t \frac{\partial}{\partial t}+\frac{x}{2} \frac{\partial}{\partial x}+\left(\frac{x^{2}}{4}+\frac{k_{3} t}{k_{1} k_{2}}\right) \frac{\partial}{\partial u}, \\
Z & =\left(u+\frac{x^{2}}{4}+\frac{k_{3} t}{k_{1} k_{2}}\right) \frac{\partial}{\partial u} .
\end{aligned}
$$

Consider the infinitesimal generator $Y$ in (3.10). The corresponding one-parameter Lie group of point transformations is obtained by solving the initial value problem for the first order system of ODEs,

$$
\frac{d x^{*}}{d \varepsilon}=\frac{x^{*}}{2},
$$




$$
\begin{gathered}
\frac{d t^{*}}{d \varepsilon}=t^{*}, \\
\frac{d u^{*}}{d \varepsilon}=\frac{\left(x^{*}\right)^{2}}{4}+\frac{k_{3} t^{*}}{k_{1} k_{2}},
\end{gathered}
$$

with $u^{*}=u, x^{*}=x, t^{*}=t$ at $\varepsilon=0$. This yields

$$
\begin{gathered}
x^{*}=X(x, t, u ; \varepsilon)=C_{3} \exp \left(\frac{\varepsilon}{2}\right), \\
t^{*}=T(x, t, u ; \varepsilon)=C_{2} \exp (\varepsilon), \\
u^{*}=U(x, t, u ; \varepsilon)=\left(\frac{C_{3}^{2}}{4}+\frac{C_{2} k_{3}}{k_{1} k_{2}}\right) \exp (\varepsilon)+C_{1} .
\end{gathered}
$$

Now we find the invariant solutions $u(x, t)$ of the model (2.1).

\section{Exact solutions}

The group invariant solutions of the laser heating CNTs formulated by using the classical heat conduction equation with various physical properties are constructed with the help of the invariant form method, direct substitution method, $\lambda$-symmetry reductions and first integrals.

\subsection{Invariant form method}

The required invariant surface condition $[10,15] \eta-\xi u_{x}-\tau u_{t}=0$ for $X$ vector field becomes

$$
u_{t}=\left(\frac{x^{2}}{4}+\frac{k_{3} t}{k_{1} k_{2}}\right) .
$$

The corresponding characteristic equations of Eq. (2.1) are given by

$$
\frac{d x}{0}=\frac{d t}{1}=\frac{d u}{\frac{x^{2}}{4}+\frac{k_{3} t}{k_{1} k_{2}}} .
$$

We yield two invariants of $X$ by solving above the characteristic equations:

$$
\zeta=x, v=u-\frac{k_{3} t^{2}}{2 k_{1} k_{2}}-\frac{x^{2}}{4} t .
$$

Hence, the solution of the invariant surface condition (4.1) is represented by the invariant form

$$
u-\frac{k_{3} t^{2}}{2 k_{1} k_{2}}-\frac{x^{2}}{4} t=\phi(x)
$$

or

$$
u=\Theta(x, t)=\frac{k_{3} t^{2}}{2 k_{1} k_{2}}+\frac{x^{2}}{4} t+\phi(\zeta),
$$

in terms of the similarity variable (one of the invariants) $\zeta=x$. Plugging of (4.5) into the classical heat conduction equation (2.1) leads to $\phi(\zeta)$ satisfying the reduced ODE which converts Eq.(2.1) to the second-order variable coefficient ODE

$$
k_{3} \phi^{\prime \prime}(\zeta)+\frac{k_{3}}{\zeta} \phi^{\prime}(\zeta)-\frac{1}{4} k_{1} k_{2} \zeta^{2}+f=0 .
$$

Thus, the invariant solution of PDE (2.1), resulting from its invariance under $X$, is presented by

$$
u=\Theta(x, t)=\frac{k_{3} t^{2}}{2 k_{1} k_{2}}+\frac{x^{2}}{4} t+\frac{1}{64} \frac{x^{4} k_{1} k_{2}}{k^{3}}-\frac{1}{4} \frac{x^{2} f}{k_{3}}+C_{1} \ln (x)+C_{2},
$$

where $C_{1}$ and $C_{2}$ are arbitrary constants.

At this point, we also note that if $\xi u_{x}+\tau u_{t}-\eta=0$ invariant surface condition is used for the $Z$ Lie vector field, then it is readily seen that $u=-\frac{x^{2}}{4}-\frac{k_{3} t}{k_{1} k_{2}}$ group invariant solution is obtained. 


\subsection{Direct substitution method}

In this alternative way $[10,15]$, we first express the invariant surface condition in a solved form $u_{t}$ (we consider the case of $Y$ Lie vector field):

$$
u_{t}=-\frac{x}{2 t} u_{x}+\frac{x^{2}}{4 t}+\frac{k_{3}}{k_{1} k_{2}} .
$$

After plugging (4.8) into heat equation (2.1), we obtain the following ODE with $t$ playing the role of a parameter:

$$
k_{3} u_{x x}+\left(k_{3} \frac{1}{x}+k_{1} k_{2} \frac{x}{2 t}\right) u_{x}-\left(\frac{x^{2}}{4 t} k_{1} k_{2}+k_{3}\right)+f=0 .
$$

The general solution of the parametric ODE (4.9) is given by

$$
u=\frac{1}{4} x^{2}-\frac{2 f t \ln (x)}{k_{1} k_{2}}-\frac{1}{2} A(t) E i\left(1, \frac{x^{2} k_{1} k_{2}}{4 k_{3} t}\right)+B(t),
$$

where $A(t)$ and $B(t)$ are arbitrary functions. Substitution of (4.10) into the invariant surface condition (4.8) yields

$$
-A^{\prime}(t) E i\left(1, \frac{x^{2} k_{1} k_{2}}{4 k_{3} t}\right) k_{1} k_{2}+2 B^{\prime}(t) k_{1} k_{2}-4 f \ln (x)-2 f-2 k_{3}=0,
$$

where the exponential integrals, $\operatorname{Ei}(a, z)$, are defined for $0<R(z)$ by

$$
\operatorname{Ei}(a, z)=\int_{1}^{\infty} e^{-k_{1} z} k_{1}^{-a} d k_{1} .
$$

We now find the one-parameter $(\varepsilon)$ family of solutions $u=\Theta(x, t ; \varepsilon)$, resulting from the invariance of the model equation (2.1) under the point symmetry $X$, obtained from any solution $u(x, t)$ that is not of the form (4.7). Let

$$
\begin{gathered}
\hat{x}=X(x, t, u ; \varepsilon)=C_{3} \exp \left(\frac{\varepsilon}{2}\right), \\
\hat{t}=T(x, t, u ; \varepsilon)=C_{2} \exp (\varepsilon), \\
\hat{u}=\Theta(\hat{x}, \hat{t}) .
\end{gathered}
$$

Then

$$
u=\Phi(x, t ; \varepsilon)=U(\hat{x}, \hat{t}, \hat{u} ;-\varepsilon)=\frac{\exp (\varepsilon)}{\frac{C_{3}^{2}}{4}+\frac{C_{2} k_{3}}{k_{1} k_{2}}} \Theta\left(C_{3} \exp \left(\frac{\varepsilon}{2}\right), C_{2} \exp (\varepsilon)\right) .
$$

\section{3 $\lambda$-Symmetry Reductions and First Integrals Using Lie Symmetry}

In this case, the Lie point symmetry generators of Eq.(4.6) are generated by the vector field of the form (see, $[21,22])$

$$
V=\xi(\zeta, \phi) \frac{\partial}{\partial \zeta}+\eta(\zeta, \phi) \frac{\partial}{\partial \phi},
$$

where $\xi$ and $\eta$ depend on $\zeta$ and $\phi$. Applying the second prolongation $p r^{(2)} V$

$$
p r^{(2)} V=X+\eta^{\zeta} \frac{\partial}{\partial \phi_{\zeta}}+\eta^{\zeta \zeta} \frac{\partial}{\partial \phi_{\zeta \zeta}}
$$

to Eq. (4.6), i.e,

$$
\left.p r^{(2)} V\left(k_{3} \phi^{\prime \prime}(\zeta)+\frac{k_{3}}{\zeta} \phi^{\prime}(\zeta)-\frac{1}{4} k_{1} k_{2} \zeta^{2}+f\right)\right|_{\phi^{\prime \prime}(\zeta)=-\frac{\phi^{\prime}(\zeta)}{\zeta}+\frac{k_{1} k_{2} \zeta^{2}}{4 k_{3}}-\frac{f}{k_{3}}}=0
$$

we get that the coefficient functions $\xi(\zeta, \phi)$ and $\eta(\zeta, \phi)$ should fulfill the following linearized symmetry equation

$$
\xi\left(-\frac{k_{3}}{\zeta^{2}} \phi_{\zeta}-\frac{1}{2} k_{1} k_{2} \zeta\right)+\frac{k_{3} \eta^{\zeta}}{\zeta}+k_{3} \eta^{\zeta \zeta}=0
$$


where $\eta^{\zeta}$ and $\eta^{\zeta \zeta}$ are the coefficients of $p r^{(2)} V$. In a simplified form these coefficients can be written in the following format:

$$
\begin{aligned}
\eta^{\zeta} & =D_{\zeta} \eta-\phi_{\zeta} D_{\zeta} \xi \\
\eta^{\zeta \zeta} & =D_{\zeta}^{2} \eta-\phi_{\zeta} D_{\zeta}^{2} \xi-2 \phi_{\zeta \zeta} D_{\zeta} \xi
\end{aligned}
$$

where $D_{\zeta}$ is the total derivatives with respect to $\zeta$ and is given as follows:

$$
D_{\zeta}=\frac{\partial}{\partial \zeta}+\phi_{\zeta} \frac{\partial}{\partial \phi}+\phi_{\zeta \zeta} \frac{\partial}{\partial \phi_{\zeta}}+\ldots
$$

If we write the prolonged coefficients (4.21) in the linearized invariance condition (4.20) and equal the coefficients of the derivatives of $\phi$ with respect to $\zeta$ to zero, we arrive at the following linear over-determined PDE system:

$$
\begin{aligned}
\eta_{\phi, \phi, \phi} & =0, \\
\eta_{\phi, \phi, \zeta} & =0 \\
\xi_{\phi, \phi} & =0 \\
\xi_{\phi, \zeta} & =\frac{\zeta \eta_{\phi, \phi}+2 \xi_{\phi}}{2 \zeta}, \\
\xi_{\zeta, \zeta} & =\frac{-3 \zeta^{4} k_{1} k_{2} \xi_{\phi}+12 f \zeta^{2} \xi_{\phi}+8 k_{3} \zeta^{2} \eta_{\phi, \zeta}+4 \zeta k_{3} \xi_{\zeta}-4 k_{3} \xi_{\zeta}}{4 \zeta^{2} k_{3}}, \\
\eta_{\zeta, \zeta} & =\frac{-k_{1} k_{2} \zeta^{3} \eta_{\phi}+2 \zeta^{3} k_{1} k_{2} \xi_{\zeta}+2 \zeta^{2} k_{1} k_{2} \xi_{\zeta}+4 f \zeta \eta_{\phi}-8 f \zeta \xi_{\zeta}-4 k_{3} \eta_{\zeta}}{4 \zeta k_{3}} .
\end{aligned}
$$

If the above over-determined system of equations is solved for $\xi(\zeta, \phi)$ and $\eta(\zeta, \phi)$, the following useful and important Lie vector fields are obtained (in fact, 6 dimensional Lie vector algebras are obtained). Hence, the point symmetry generators admitted by the equation (4.6) are given by

$$
\begin{aligned}
V_{1}= & \frac{\partial}{\partial \phi}, \\
V_{2}= & \ln (\zeta) \frac{\partial}{\partial \phi}, \\
V_{3}= & 4 k_{3} \zeta \frac{\partial}{\partial \zeta}-\frac{\zeta^{2}\left(-k_{1} k_{2}^{2} \zeta+8 f\right)}{4} \frac{\partial}{\partial \phi} \\
V_{4}= & (16 \zeta \ln (\zeta)-8 \zeta) k_{1} k_{2} k_{3} \frac{\partial}{\partial \zeta}+\left(k_{1}^{2} k_{2}^{2} \zeta^{4} \ln (\zeta)\right. \\
& \left.-\frac{k_{1}^{2} k_{2}^{2} \zeta^{4}}{2}-8 k_{1} k_{2} f \zeta^{2} \ln (\zeta)+4 f k_{1} k_{2} \zeta^{2}+8 f^{2} \ln (\zeta)\right) \frac{\partial}{\partial \phi} .
\end{aligned}
$$

Now we use the relationship between Lie point symmetries and $\lambda$-symmetries to get $\lambda$-symmetries of Equation (4.6). Let us consider $X_{3}$. Then we have

$$
\begin{aligned}
\xi & =4 k_{3} \zeta \\
\eta & =-\frac{\zeta^{2}\left(-k_{1} k_{2}^{2} \zeta+8 f\right)}{4}
\end{aligned}
$$


and the characteristic function of $V_{3}$

$$
\begin{aligned}
Q & =\eta-\xi \phi_{\zeta} \\
& =-\frac{\zeta^{2}\left(-k_{1} k_{2}^{2} \zeta+8 f\right)}{4}-4 k_{3} \zeta \phi_{\zeta},
\end{aligned}
$$

and the total derivative operator

$$
D_{\zeta}=\frac{\partial}{\partial \zeta}+\phi_{\zeta} \frac{\partial}{\partial \phi}+\phi_{\zeta \zeta} \frac{\partial}{\partial \phi_{\zeta}}=\frac{\partial}{\partial \zeta}+\phi_{\zeta} \frac{\partial}{\partial \phi}+\left(-\frac{\phi_{\zeta}}{\zeta}+\frac{k_{1} k_{2} \zeta^{2}}{4 k_{3}}-\frac{f}{k_{3}}\right) \frac{\partial}{\partial \phi_{\zeta}} .
$$

The symmetry $v=\frac{\partial}{\partial \phi}$ is the $\lambda$-symmetry when

$$
\lambda=\frac{D_{\zeta}(Q)}{Q}=-\frac{\zeta k_{1} k_{2}\left(-3 k_{2}+4 \zeta\right)}{\zeta^{2} k_{1} k_{2}^{2}-8 \zeta f-16 k_{3} \phi_{\zeta}} .
$$

In this stage, we wish to calculate a first integral from $\lambda$. Plugging Eq. (4.29) into

$$
\omega_{\phi}+\lambda \omega_{\phi_{\zeta}}=0
$$

we obtain

$$
\omega_{\phi}-\frac{\zeta k_{1} k_{2}\left(-3 k_{2}+4 \zeta\right)}{\zeta^{2} k_{1} k_{2}^{2}-8 \zeta f-16 k_{3} \phi_{\zeta}} \omega_{\phi_{\zeta}}=0 .
$$

Integrating the characteristic equation of (4.31)

$$
\frac{d \phi}{1}=-\frac{\left(\zeta^{2} k_{1} k_{2}^{2}-8 \zeta f-16 k_{3} \phi_{\zeta}\right) d \phi_{\zeta}}{\zeta k_{1} k_{2}\left(-3 k_{2}+4 \zeta\right)},
$$

we deduce a special solution

$$
\omega\left(\zeta, \phi, \phi_{\zeta}\right)=\frac{-\zeta^{2} \phi_{\zeta} k_{1}{k_{2}}^{2}-4 \zeta^{2} \phi k_{1} k_{2}+3 \zeta \phi k_{1}{k_{2}}^{2}+8 \zeta \phi_{\zeta} f+8 \phi_{\zeta}^{2} k_{3}}{\zeta k_{1} k_{2}\left(-3 k_{2}+4 \zeta\right)} .
$$

Secondly, calculating function $D[\omega]$, one can get

$$
\begin{aligned}
D[\omega]= & \frac{\partial \omega}{\partial \zeta}+\phi_{\zeta} \frac{\partial \omega}{\partial \phi}+\left(-\frac{\phi_{\zeta}}{\zeta}+\frac{k_{1} k_{2} \zeta^{2}}{4 k_{3}}-\frac{f}{k_{3}}\right) \frac{\partial \omega}{\partial \phi_{\zeta}} \\
= & -\frac{1}{4 \zeta^{2} k_{1} k_{2}\left(-3 k_{2}+4 \zeta\right)^{2} k_{3}}\left(4 \zeta^{6}{k_{1}}^{2}{k_{2}}^{3}-3 \zeta^{5}{k_{1}}^{2} k_{2}{ }^{4}\right. \\
& -32 \zeta^{5} f k_{1} k_{2}+8 \zeta^{4} f k_{1}{k_{2}}^{2}-64 \zeta^{3} \phi_{\zeta} k_{1} k_{2}{ }^{2} k_{3}+12 \zeta^{3} f k_{1} k_{2}{ }^{3} \\
& +36 \zeta^{2} \phi_{\zeta} k_{1} k_{2}{ }^{3} k_{3}+128 \zeta^{3} f^{2}+512 \zeta^{2} \phi_{\zeta} f k_{3}-96 \zeta^{2} f^{2} k_{2} \\
& \left.+512 \zeta \phi_{\zeta}^{2} k_{3}{ }^{2}-288 \zeta \phi_{\zeta} f k_{2} k_{3}-288 \phi_{\zeta}^{2} k_{2} k_{3}{ }^{2}\right) \\
= & -\frac{1}{4 \zeta k_{3}\left(-3 k_{2}+4 \zeta\right) k_{1} k_{2}}\left(k_{1}{ }^{2} k_{2}{ }^{3} \zeta^{4}-8 f k_{1} k_{2} \zeta^{3}-4 \zeta^{2} f k_{1} k_{2}{ }^{2}\right. \\
& \left.+64 \zeta \phi k_{1} k_{2} k_{3}+64 \zeta \omega k_{1} k_{2} k_{3}-36 \phi k_{1} k_{2}{ }^{2} k_{3}-36 \omega k_{1} k_{2}{ }^{2} k_{3}+32 \zeta f^{2}\right) \\
= & F(\zeta, \phi, \omega) .
\end{aligned}
$$


Next, calculating the first-order partial differential equation

$$
G_{\zeta}+\phi_{\zeta} G_{\phi}-\frac{\left(\begin{array}{c}
k_{1}{ }^{2} k_{2}{ }^{3} \zeta^{4}-8 f k_{1} k_{2} \zeta^{3}-4 \zeta^{2} f k_{1} k_{2}{ }^{2}+64 \zeta \phi k_{1} k_{2} k_{3} \\
+64 \zeta \omega k_{1} k_{2} k_{3}-36 \phi k_{1} k_{2}{ }^{2} k_{3}-36 \omega k_{1} k_{2}{ }^{2} k_{3}+32 \zeta f^{2}
\end{array}\right) G_{\omega}}{4 \zeta k_{3}\left(-3 k_{2}+4 \zeta\right) k_{1} k_{2}}=0
$$

and solving the corresponding characteristic equation, we get a special solution

$$
\begin{aligned}
G\left(\zeta, \phi, \phi_{\zeta}, \omega\right)= & \frac{\zeta^{3}}{420 k_{1} k_{2} k_{3}}\left(15 k_{1}{ }^{2}{k_{2}}^{3} \zeta^{4}+1344 \phi_{\zeta} \zeta^{2} k_{1} k_{2} k_{3}-945 \phi_{\zeta} \zeta k_{1} k_{2}{ }^{2} k_{3}\right. \\
& -140 f k_{1} k_{2} \zeta^{3}-84 \zeta^{2} f k_{1}{k_{2}}^{2}+1680\left(-\zeta \phi_{\zeta}+\phi\right) \zeta k_{1} k_{2} k_{3} \\
& \left.-1260\left(-\zeta \phi_{\zeta}+\phi\right) k_{1}{k_{2}}^{2} k_{3}+1680 \zeta w k_{1} k_{2} k_{3}-1260 w k_{1} k_{2}{ }^{2} k_{3}+840 \zeta f^{2}\right) .
\end{aligned}
$$

Finally, substituting (4.33) into (4.39), we get the first integral

$$
I=\frac{\zeta^{2}\left(\begin{array}{c}
15 \zeta^{5} k_{1}{ }^{2} k_{2}{ }^{3}-140 \zeta^{4} f k_{1} k_{2}-336 \phi_{\zeta} \zeta^{3} k_{1} k_{2} k_{3}-84 \zeta^{3} f k_{1} k_{2}{ }^{2} \\
-105 \phi_{\zeta} \zeta^{2} k_{1} k_{2}{ }^{2} k_{3}+840 \zeta^{2} f^{2}+3360 \phi_{\zeta} f k_{3} \zeta+3360 \phi_{\zeta}^{2} k_{3}{ }^{2}
\end{array}\right)}{420 k_{1} k_{2} k_{3}} .
$$

We get the invariant solution of Eq. (4.6) by integrating Eq. (4.40) as follows

$$
\phi(\zeta)=\int \frac{P(\zeta)}{6720 k_{3}} d \zeta+C_{1}
$$

where

$$
\begin{aligned}
P(\zeta)= & 336 \zeta^{3} k_{1} k_{2}+105 \zeta^{2} k_{1}{k_{2}}^{2}-3360 f \zeta \\
& \pm \zeta \sqrt{-21 \zeta k_{1} k_{2}\left(-5376 \zeta^{3} k_{1} k_{2}+6240 \zeta^{2} k_{1}{k_{2}}^{2}-525 \zeta k_{1}{k_{2}}^{3}+17920 f \zeta-20160 f k_{2}\right)} .
\end{aligned}
$$

Similarly, we get the invariant solution of Eq. (2.1) by using Eq. (4.5) as follows

$$
u(x, t)=\frac{k_{3} t^{2}}{2 k_{1} k_{2}}+\frac{x^{2}}{4} t+\int \frac{P(x)}{6720 k_{3}} d x+C_{1},
$$

where

$$
\begin{aligned}
P(x)= & 336 x^{3} k_{1} k_{2}+105 x^{2} k_{1}{k_{2}}^{2}-3360 f x \\
& \pm x \sqrt{-21 x k_{1} k_{2}\left(-5376 x^{3} k_{1} k_{2}+6240 x^{2} k_{1}{k_{2}}^{2}-525 x k_{1} k_{2}{ }^{3}+17920 f x-20160 f k_{2}\right)} .
\end{aligned}
$$

\section{Conservation laws of Eq.(2.1)}

In order to produce conserved vectors, we perform multiplier approach [14], [23] depend upon on the famous result that the Euler-Lagrange operator annihilates a total divergence. Firstly, if $\left(T^{t}, T^{x}\right)$ is a conserved vector related with a conservation law, then

$$
D_{t} T^{t}+D_{x} T^{x}=0
$$

on the solutions of Eq. (2.1). Furthermore, if there exists a nontrivial differential function $\Lambda$, defined a multiplier or characteristic function such that $E_{u}(\Lambda G)=0$, then $\Lambda G$ is a total divergence, i.e., $\Lambda G=D_{t} T^{t}+D_{x} T^{x}$ for some (conserved) vector $\left(T^{t}, T^{x}\right)$ and $E_{u}$ is the respective Euler-Lagrange operator. Thus, knowledge of each multiplier $\Lambda\left(x, t, u, u_{x}\right)$ leads to conserved vectors computed by a homotopy operator [14, 15, 23]. For Eq. (2.1), we obtain the multipliers, $\Lambda_{1}, \Lambda_{2}$, that are given by

$$
\Lambda_{1}\left(x, t, u, u_{x}, u_{t}\right)=\frac{x J_{0}\left(\sqrt{-c_{1}} x\right)}{\mathrm{e}^{\frac{k_{3} c_{1} t}{k_{1} k_{2}}}}
$$



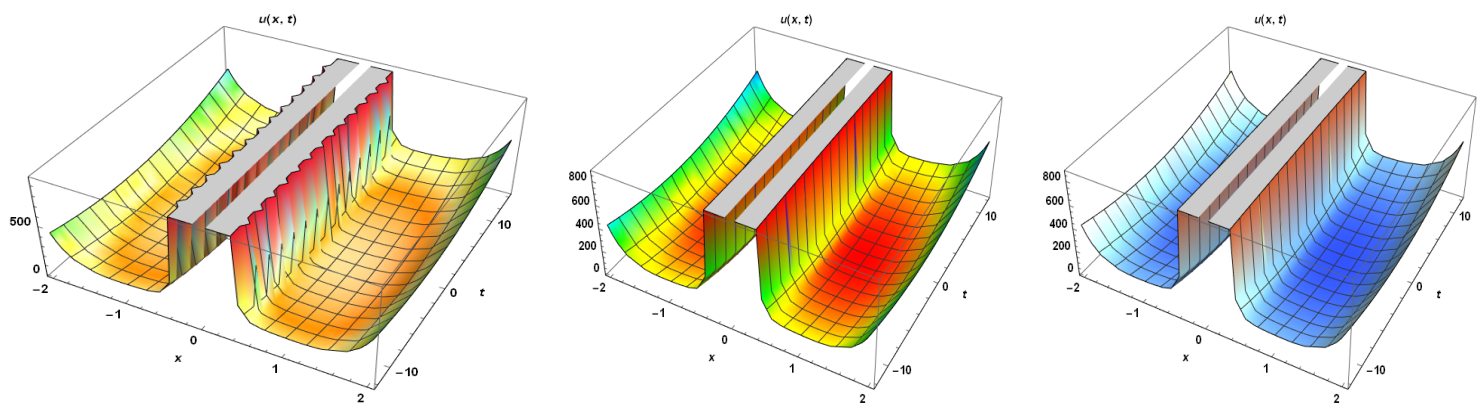

Figure 1. Three-dimensional plots of the solution (4.7) setting all arbitrary parameters to unity.
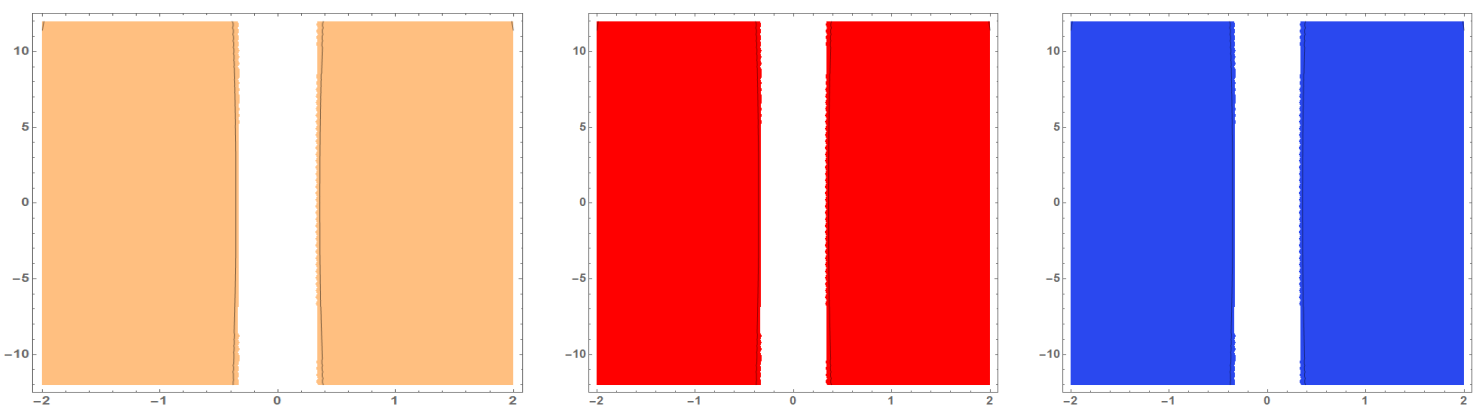

Figure 2. Contour plots of the solution (4.7) setting all arbitrary parameters to unity.

$$
\Lambda_{2}\left(x, t, u, u_{x}, u_{t}\right)=\frac{x Y_{0}\left(\sqrt{-c_{1}} x\right)}{\mathrm{e}^{\frac{k_{3} c_{1} t}{k_{1} k_{2}}}},
$$

where $J_{0}$ and $Y_{0}$ are the first and second kind Bessel functions, respectively. Thus, corresponding to the above multipliers we have the following conservation laws of Eq. (2.1):

$$
\begin{gathered}
T^{x}=\frac{1}{f}\left(\begin{array}{c}
-f\left(C_{2} u+x C_{4}+C_{3} t+C_{13}\right) u_{t}-2 x u_{x}\left(C_{10}+\frac{C_{6}}{2}\right) k_{3} \\
+\left(-u C_{3}+C_{10} x^{2}+\left(-2 C_{5} t-C_{14}\right) x+C_{11} t^{3}+C_{9} t^{2}+C_{8} t+C_{1}\right) f
\end{array}\right), \\
T^{t}=\frac{1}{f}\left(\begin{array}{c}
f\left(C_{2} u+x C_{4}+C_{3} t+C_{13}\right) u_{x}+\left(C_{4} f+2 k_{1} x k_{2}\left(C_{10}+\frac{C_{6}}{2}\right)\right) u \\
+\left(C_{7} x^{2}+\left(C_{6} t+C_{15}\right) x+C_{6} t^{2}+C_{14} t+C_{12}\right) f
\end{array}\right) .
\end{gathered}
$$

\section{Conclusion}

In this study, laser heated CNT equation used in cancer research has been discussed and Lie group analysis has been applied in detail. In this sense, group-invariant solutions i.e, Eqs. (4.7), (4.10) and (4.43) were obtained. The accuracy of the solutions obtained has been tested and verified in Maple program. The obtained analytical solutions have been established for the first time in the literature and can be used in experimental research for cancer studies. It may also be useful in the production of suitable carbon nanotubes that can be used in later stages. Besides, local
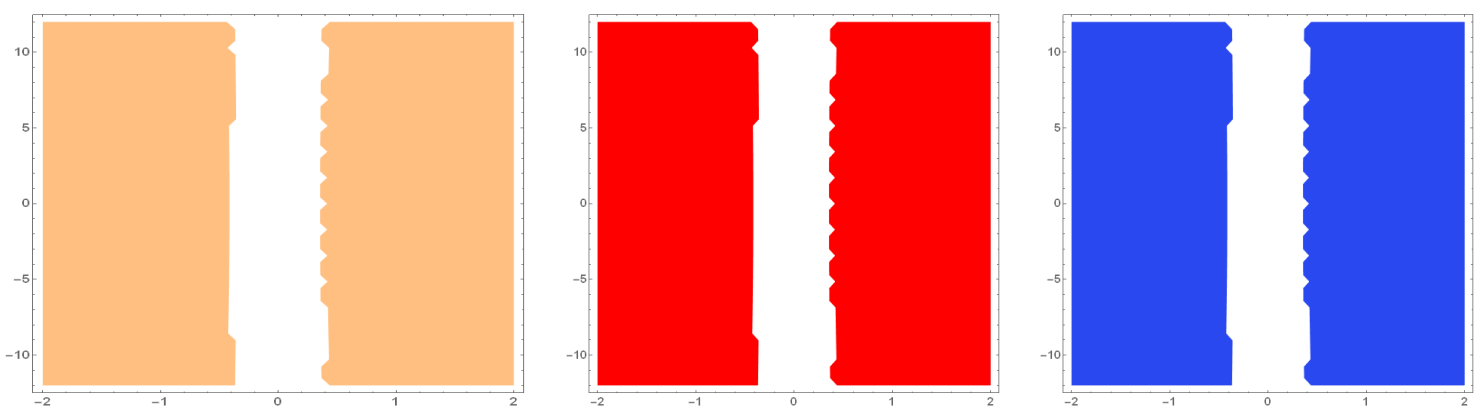

Figure 3. Density plots of the solution (4.7) setting all arbitrary parameters to unity. 

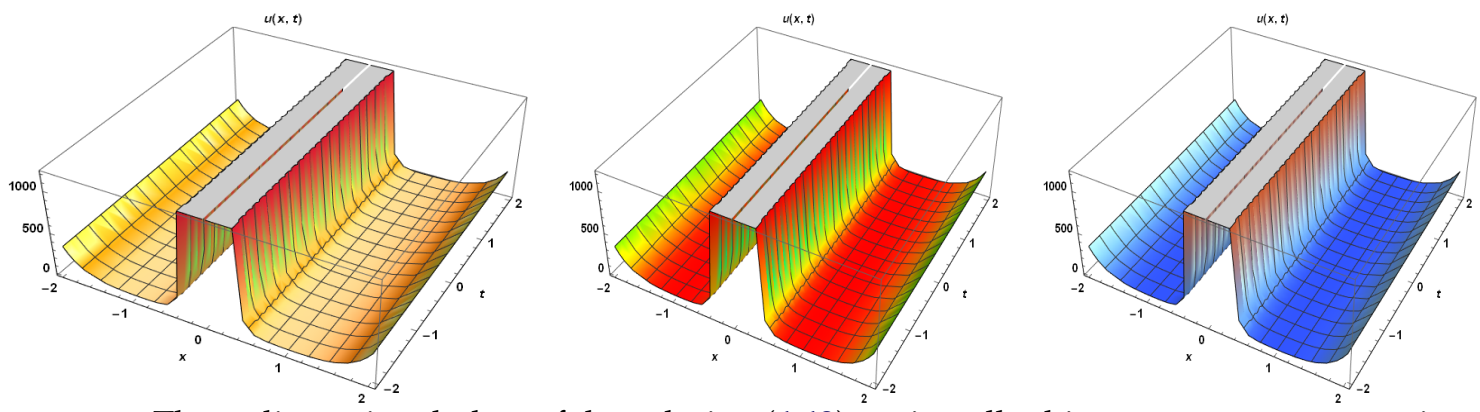

Figure 4. Three-dimensional plots of the solution (4.43) setting all arbitrary parameters to unity.
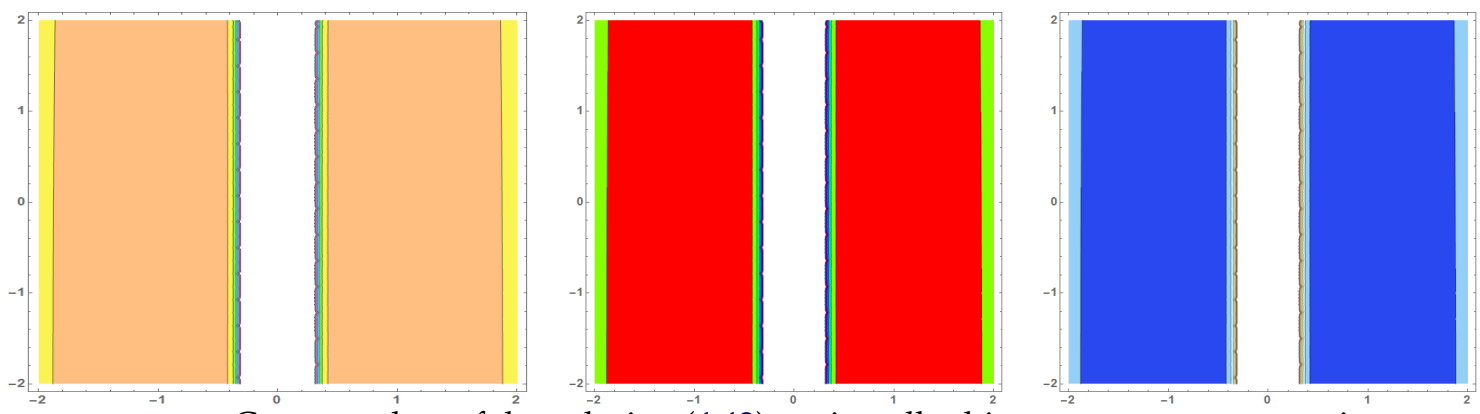

Figure 5. Contour plots of the solution (4.43) setting all arbitrary parameters to unity.
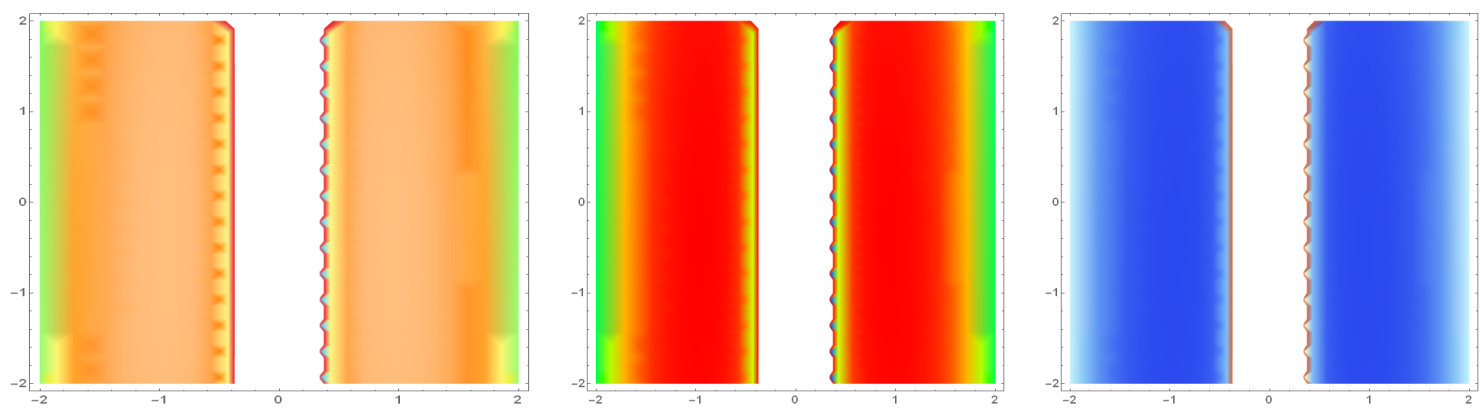

Figure 6. Density plots of the solution (4.43) setting all arbitrary parameters to unity. 
conservation laws of the model were obtained. In addition, an open problem for the model is the $\mu$-symmetry concept [24], which is a new type of symmetry, and these open questions will be addressed in future studies.

\section{Acknowledgment.}

This study was supported by Scientific Research Project Unit (BAP) of Bursa Uludağ University, under project number: KUAP(F)-2019/11. The authors thank to Bursa Uludag University.

\section{Funding}

There is no funding for this work.

\section{Availability of data and materials}

Not applicable.

\section{Competing interests}

The authors declare that they have no competing interests.

\section{Author's contributions}

All authors contributed equally to the writing of this paper. All authors read and approved the final manuscript.

\section{References}

[1] Yaşar, E., Yıldırım, Y., \& Khalique, C. M.: Lie symmetry analysis, conservation laws and exact solutions of the seventh-order time fractional Sawada-Kotera-Ito equation. Results in physics. 6, 322-328 (2016).

[2] Yaşar, E., Yıldırım, Y., \& Adem, A. R.: Perturbed optical solitons with spatio-temporal dispersion in (2+ 1)-dimensions by extended Kudryashov method. Optik. 158, 1-14 (2018).

[3] Yaşar, E.: On the conservation laws and invariant solutions of the $m K d V$ equation. Journal of mathematical analysis and applications, 363(1), 174-181 (2010).

[4] Yaşar, E., \& Özer, T.: On symmetries, conservation laws and invariant solutions of the foam-drainage equation. International Journal of Non-Linear Mechanics, 46(2), 357-362 (2011).

[5] Yaşar, E., Yıldırım, Y., \& Adem, A. R.: Extended transformed rational function method to nonlinear evolution equations. International Journal of Nonlinear Sciences and Numerical Simulation, 20(6), 691-701 (2019).

[6] Hirota, R.: The direct method in soliton theory (No. 155). Cambridge University Press (2004).

[7] Fuchssteiner, B., \& Fokas, A. S.: Symplectic structures, their Bäcklund transformations and hereditary symmetries. Physica D: Nonlinear Phenomena, 4(1), 47-66 (1981).

[8] Guo, B., Ling, L., \& Liu, Q. P.: Nonlinear Schrödinger equation: generalized Darboux transformation and rogue wave solutions. Physical Review E, 85(2), 026607 (2012).

[9] Ramani, A., Grammaticos, B., \& Bountis, T.: The Painlevé property and singularity analysis of integrable and non-integrable systems. Physics Reports, 180(3), 159-245 (1989).

[10] Bluman, G., \& Anco, S.: Symmetry and integration methods for differential equations (Vol. 154). Springer Science \& Business Media (2008).

[11] He, J. H.: Variational iteration method-a kind of non-linear analytical technique: some examples. International journal of non-linear mechanics, 34(4), 699-708 (1999). 
[12] Malfliet, W., \& Hereman, W.: The tanh method: I. Exact solutions of nonlinear evolution and wave equations. Physica Scripta, 54(6), 563 (1996).

[13] Ma, W. X.: A refined invariant subspace method and applications to evolution equations. Science China Mathematics, 55(9), 1769-1778 (2012).

[14] Olver, P. J.: Applications of Lie groups to differential equations (Vol. 107). Springer Science \& Business Media (2000).

[15] Bluman, G. W., Cheviakov, A. F., \& Anco, S. C.: Applications of symmetry methods to partial differential equations (Vol. 168, pp. xx+-398). New York: Springer (2010).

[16] Siregar, S., Oktamuliani, S., \& Saijo, Y.: A theoretical model of laser heating carbon nanotubes. Nanomaterials, 8(8), 580 (2018).

[17] Nakamiya, T., Ueda, T., Ikegami, T., Ebihara, K., \& Tsuda, R.: Thermal analysis of carbon nanotube film irradiated by a pulsed laser. Current Applied Physics, 8(3-4), 400-403(2008).

[18] Nakamiya, T., \& Ebihara, K.: The Finite Element Thermal Analysis of Amorphous Silicon Thin Films Irradiated by a Pulsed Laser. The transactions of the Institute of Electrical Engineers of Japan. A, 108(10), 443-450 (1988).

[19] Younis, M., \& Rizvi, S. T. R.: Optical soliton like-pulses in ring-cavity fiber lasers of carbon nanotubes. Journal of Nanoelectronics and Optoelectronics, 11(3), 276-279 (2016).

[20] Wang, G., Kara, A. H., Buhe, E., \& Fakhar, K.: Group analysis and conservation laws of a coupled system of partial differential equations describing the carbon nanotubes conveying fluid. Romanian Journal in physics, 60(7-8), 952-960 (2015).

[21] Muriel, C.; Romero, J.L.: First integrals, integrating factors and $\lambda$-Symmetries of second-order differential equations. J. Phys. A Math. Theor. 42, 365207 (2009).

[22] Bai, Y. S., Pei, J. T., \& Ma, W. X.: Symmetry and-Symmetry Reductions and Invariant Solutions of Four Nonlinear Differential Equations. Mathematics, 8, 1138 (2020).

[23] A. Cheviakov.: GeM software package for computation of symmetries and conservation laws of differential equations. Comp. Phys. Comm. 176 (2007), 48-61.

[24] Gaeta, G. C., \& Morando, P.: On the relation between standard and $\mu$-symmetries for PDEs. J. Phys. A: Math. Gen., Vol. 37, 9467-9486 (2004).

\section{Affiliations}

EMRULLAH YAŞAR

ADDRESS: Department of Mathematics, Faculty of Arts and Sciences, Bursa Uludag University, 16059 Bursa, Turkey

E-MAIL: eyasar@uludag.edu.tr

ORCID ID:0000-0003-4732-5753

YAKUP YILDIRIM

ADDRESS: Department of Mathematics, Faculty of Arts and Sciences, Near East University, 99138 Nicosia, Cyprus

E-MAIL: yakup.yildirim@neu.edu.tr

ORCID ID:0000-0003-4443-3337 